\title{
REPDIGITS AS SUMS OF THREE BALANCING NUMBERS
}

\author{
MAHADI DDAMULIRA
}

\begin{abstract}
Let $\left\{B_{n}\right\}_{n>0}$ be the sequence of Balancing numbers defined by $B_{0}=0, B_{1}=1$, and $B_{n+2}=6 B_{n+1}-B_{n}$ for all $n \geq 0$. In this paper, we find all repdigits in base 10 which can
\end{abstract} be written as a sum of three Balancing numbers.

\section{INTRODUCTION}

The concept of balancing numbers was first introduced by Bahera and Panda [1 in connection with the Diophantine equation that consists of finding a natural number $n$ such that

$$
1+2+\cdots+(n-1)=(n+1)+(n+2)+\cdots+(n+r),
$$

for some natural number $r$. The number $n$ is called the balancing number and the number $r$ is called the balancer corresponding to $n$. For example 6,35, and 204 are balacing numbers with balancers 2,14 , and 84 , respectively.

We can also define the balancing numbers as a recurrence sequence. Let $\left\{B_{n}\right\}_{n \geq 0}$ be the sequence of balancing numbers given by

$$
B_{0}=0, B_{1}=1 \text {, and } B_{n+2}=6 B_{n+1}-B_{n} \text { for all } n \geq 0 .
$$

This is sequence $A 001109$ on the On-Line Encyclopedia of Integer Sequences (OEIS) [10]. The first few terms of this sequence are

$$
\left\{B_{n}\right\}_{n \geq 0}=0,1,6,35,204,1189,6930,40391,235416,1372105,7997214,46611179, \ldots
$$

The Binet formula for the general terms of this sequence is given by

$$
B_{n}=\frac{\gamma^{n}-\delta^{n}}{4 \sqrt{2}}
$$

where $(\gamma, \delta)=(3+2 \sqrt{2}, 3-2 \sqrt{2})$ are the roots of $x^{2}-6 x+1=0$, which is the characteristic equation of the Balancing sequence.

Furthermore, it can be noted that $5<\gamma<6,0<\delta<1$ and we can prove by induction that

$$
\gamma^{n-1} \leq B_{n}<\gamma^{n},
$$

holds for all $n \geq 1$.

A repdigit is a positive integer $N$ that has only one distinct digit when written in base 10. That is, $N$ is of the form

$$
N=d\left(\frac{10^{\ell}-1}{9}\right)
$$

2010 Mathematics Subject Classification. Primary 11B39, 11D45; Secondary 11D61, 11J86.

Key words and phrases. Balancing number, repdigit, linear form in logarithms, reduction method.

This research was supported by the Austrian Science Fund (FWF) projects: F5510-N26 - Part of the special research program (SFB), "Quasi-Monte Carlo Methods: Theory and Applications" and W1230 - "Doctoral Program Discrete Mathematics". 
for some positive integers $d, \ell$ with $1 \leq d \leq 9$. The sequence of repdigits is sequence $A 010785$ on the OEIS.

\section{Main Result}

In this paper, we study the problem of writing repdigits as sums of three balancing numbers. More precisely, we completely solve the Diophantine equation

$$
N=B_{n_{1}}+B_{n_{2}}+B_{n_{3}}=d\left(\frac{10^{\ell}-1}{9}\right),
$$

in non-negative integers $\left(N, n_{1}, n_{2}, n_{3}, d, \ell\right)$ with $n_{1} \geq n_{2} \geq n_{3} \geq 0, \ell \geq 1$, and $1 \leq d \leq 9$.

Our main result is the following.

Theorem 2.1. All non-negative integer solutions $\left(N, n_{1}, n_{2}, n_{3}, d, \ell\right)$ of the Diophantine equation (2.1) with $n_{1} \geq n_{2} \geq n_{3} \geq 0, \ell \geq 1$, and $1 \leq d \leq 9$, arise from

$$
N \in\{1,2,3,6,7,8\} \text {. }
$$

This paper serves as a continuation of the results in [5, 6, 7, 9]. The method of proof involves the application of Baker's theory for linear forms in logarithms of algebraic numbers, and the Baker-Davenport reduction procedure. Computations are done with the help of a simple computer program in Mathematica.

\section{Preliminary Results}

3.1. Linear forms in logarithms. Let $\eta$ be an algebraic number of degree $d$ with minimal primitive polynomial over the integers

$$
a_{0} x^{d}+a_{1} x^{d-1}+\cdots+a_{d}=a_{0} \prod_{i=1}^{d}\left(x-\eta^{(i)}\right),
$$

where the leading coefficient $a_{0}$ is positive and the $\eta^{(i)}$ 's are the conjugates of $\eta$. Then the $\log a$ rithmic height of $\eta$ is given by

$$
h(\eta):=\frac{1}{d}\left(\log a_{0}+\sum_{i=1}^{d} \log \left(\max \left\{\left|\eta^{(i)}\right|, 1\right\}\right)\right) .
$$

In particular, if $\eta=p / q$ is a rational number with $\operatorname{gcd}(p, q)=1$ and $q>0$, then $h(\eta)=$ $\log \max \{|p|, q\}$. The following are some of the properties of the logarithmic height function $h(\cdot)$, which will be used in the next sections of this paper without reference:

$$
\begin{aligned}
h\left(\eta_{1} \pm \eta_{2}\right) & \leq h\left(\eta_{1}\right)+h\left(\eta_{2}\right)+\log 2, \\
h\left(\eta_{1} \eta_{2}^{ \pm 1}\right) & \leq h\left(\eta_{1}\right)+h\left(\eta_{2}\right), \\
h\left(\eta^{s}\right) & =|s| h(\eta)(s \in \mathbb{Z}) .
\end{aligned}
$$

We recall the result of Bugeaud, Mignotte, and Siksek (2], Theorem 9.4, pp. 989), which is a modified version of the result of Matveev [8], which is one of our main tools in this paper.

Theorem 3.1. Let $\eta_{1}, \ldots, \eta_{t}$ be positive real algebraic numbers in a real algebraic number field $\mathbb{K} \subset \mathbb{R}$ of degree $D_{\mathbb{K}}, b_{1}, \ldots, b_{t}$ be nonzero integers, and assume that

$$
\Lambda:=\eta_{1}^{b_{1}} \cdots \eta_{t}^{b_{t}}-1
$$

is nonzero. Then

$$
\log |\Lambda|>-1.4 \times 30^{t+3} \times t^{4.5} \times D_{\mathbb{K}}^{2}\left(1+\log D_{\mathbb{K}}\right)(1+\log B) A_{1} \cdots A_{t},
$$


where

and

$$
B \geq \max \left\{\left|b_{1}\right|, \ldots,\left|b_{t}\right|\right\}
$$

$$
A_{i} \geq \max \left\{D_{\mathbb{K}} h\left(\eta_{i}\right),\left|\log \eta_{i}\right|, 0.16\right\}, \quad \text { for all } \quad i=1, \ldots, t .
$$

3.2. Reduction procedure. During the calculations, we get upper bounds on our variables which are too large, thus we need to reduce them. To do so, we use some result from the theory of continued fractions.

For a nonhomogeneous linear form in two integer variables, we use a slight variation of a result due to Dujella and Pethő (see [3], Lemma 5a). For a real number $X$, we write $\|X\|:=\min \{|X-n|$ : $n \in \mathbb{Z}\}$ for the distance from $X$ to the nearest integer.

Lemma 3.1. Let $M$ be a positive integer, $\frac{p}{q}$ be a convergent of the continued fraction expansion of the irrational number $\tau$ such that $q>6 M$, and $A, B, \mu$ be some real numbers with $A>0$ and $B>1$. Furthermore, let $\varepsilon:=\|\mu q\|-M\|\tau q\|$. If $\varepsilon>0$, then there is no solution to the inequality

$$
0<|u \tau-v+\mu|<A B^{-w},
$$

in positive integers $u, v$, and $w$ with

$$
u \leq M \quad \text { and } \quad w \geq \frac{\log (A q / \varepsilon)}{\log B} .
$$

Finally, the following Lemma is also useful. It is Lemma 7 in [4].

Lemma 3.2. If $r \geq 1, H>\left(4 r^{2}\right)^{r}$, and $H>L /(\log L)^{r}$, then

$$
L<2^{r} H(\log H)^{r} .
$$

\section{BOUNDing THE VARIABLES}

We assume that $n_{1} \geq n_{2} \geq n_{3}$. From 1.2 and 2.1, we have

$$
\gamma^{n_{1}-1} \leq B_{n_{1}} \leq B_{n_{1}}+B_{n_{2}}+B_{n_{3}}=d\left(\frac{10^{\ell}-1}{9}\right) \leq 10^{\ell}
$$

and

$$
10^{\ell-1} \leq d\left(\frac{10^{\ell}-1}{9}\right)=B_{n_{1}}+B_{n_{2}}+B_{n_{3}} \leq 3 B_{n_{1}}<\gamma^{n_{1}+1}
$$

where we use $\gamma>3$. Thus,

$$
\left(n_{1}-1\right) \frac{\log \gamma}{\log 10} \leq \ell \quad \text { and } \quad \ell-1 \leq\left(n_{1}+1\right) \frac{\log \gamma}{\log 10} .
$$

Since $\log \gamma / \log 10=0.76555 . .<4 / 5$, we can conclude from the above that

$$
\frac{3}{5}\left(n_{1}-1\right)<\ell<1+\frac{4}{5}\left(n_{1}+1\right) .
$$

Running a Mathematica program in the range $0 \leq n_{3} \leq n_{2} \leq n_{1} \leq 100,1 \leq d \leq 9$, and $1 \leq \ell \leq 100$ we obtain only the solutions listed in Theorem 2.1. From now onwards, we assume that $n_{1}>100$.

Equation (2.1) can be written as

$$
\frac{\gamma^{n_{1}}-\delta^{n_{1}}}{4 \sqrt{2}}+\frac{\gamma^{n_{2}}-\delta^{n_{2}}}{4 \sqrt{2}}+\frac{\gamma^{n_{3}}-\delta^{n_{3}}}{4 \sqrt{2}}=d\left(\frac{10^{\ell}-1}{9}\right) .
$$

Then, we rewrite 4.2 in three ways and apply lower bounds for linear forms in logarithms in three steps as follows: 
4.1. Step 1. We have that

$$
\frac{\gamma^{n_{1}}-\delta^{n_{1}}}{4 \sqrt{2}}+\frac{\gamma^{n_{2}}-\delta^{n_{2}}}{4 \sqrt{2}}+\frac{\gamma^{n_{3}}-\delta^{n_{3}}}{4 \sqrt{2}}-\frac{d \cdot 10^{\ell}}{9}=-\frac{d}{9} .
$$

This is equivalent to

$$
\frac{\gamma^{n_{1}}}{4 \sqrt{2}}\left(1+\gamma^{n_{2}-n_{1}}+\gamma^{n_{3}-n_{1}}\right)-\frac{d \cdot 10^{\ell}}{9}=-\frac{d}{9}+\frac{1}{4 \sqrt{2}}\left(\delta^{n_{1}}+\delta^{n_{2}}+\delta^{n_{3}}\right) .
$$

Thus, we have

$$
\begin{aligned}
\left|\frac{\gamma^{n_{1}}}{4 \sqrt{2}}\left(1+\gamma^{n_{2}-n_{1}}+\gamma^{n_{3}-n_{1}}\right)-\frac{d \cdot 10^{\ell}}{9}\right| & \leq \frac{d}{9}+\frac{1}{4 \sqrt{2}}\left(\delta^{n_{1}}+\delta^{n_{2}}+\delta^{n_{3}}\right) \\
& <\frac{1}{4 \sqrt{2}}(4 \sqrt{2}+3),
\end{aligned}
$$

and so

$$
\left|\frac{\gamma^{n_{1}}}{4 \sqrt{2}}\left(1+\gamma^{n_{2}-n_{1}}+\gamma^{n_{3}-n_{1}}\right)-\frac{d \cdot 10^{\ell}}{9}\right|<\frac{\gamma}{2 \sqrt{2}} .
$$

We divide through 4.3 by $\alpha^{n_{1}}\left(1+\gamma^{n_{2}-n_{1}}+\gamma^{n_{3}-n_{1}}\right) /(4 \sqrt{2})$ to get

$$
\left|10^{\ell} \gamma^{-n_{3}}\left(\frac{4 d \sqrt{2}}{9\left(1+\gamma^{n_{1}-n_{3}}+\gamma^{n_{2}-n_{3}}\right)}\right)-1\right|<\frac{2 \gamma^{1-n_{1}}}{1+\gamma^{n_{2}-n_{1}}+\gamma^{n_{3}-n_{1}}}<\gamma^{2-n_{1}} .
$$

Thus, we have

$$
\left|10^{\ell} \gamma^{-n_{3}}\left(\frac{4 d \sqrt{2}}{9\left(1+\gamma^{n_{1}-n_{3}}+\gamma^{n_{2}-n_{3}}\right)}\right)-1\right|<\gamma^{2-n_{1}}
$$

We put

$$
\Lambda_{1}:=10^{\ell} \gamma^{-n_{3}}\left(\frac{4 d \sqrt{2}}{9\left(1+\gamma^{n_{1}-n_{3}}+\gamma^{n_{2}-n_{3}}\right)}\right)-1 .
$$

In order to apply Theorem 3.1 we need to check that $\Lambda_{1} \neq 0$. Suppose that $\Lambda_{1}=0$, then we have

$$
\gamma^{n_{1}}+\gamma^{n_{2}}+\gamma^{n_{3}}=\frac{10^{\ell} \cdot 4 d \sqrt{2}}{9}
$$

By conjugating the above relation in $\mathbb{Q}(\sqrt{2})$, we get

$$
\delta^{n_{1}}+\delta^{n_{2}}+\delta^{n_{3}}=-\frac{10^{\ell} \cdot 4 d \sqrt{2}}{9} .
$$

Taking absolute values on both sides of the above equation, we obtain that

$$
\frac{10^{\ell} \cdot 4 d \sqrt{2}}{9}=\left|\delta^{n_{1}}+\delta^{n_{2}}+\delta^{n_{3}}\right| \leq|\delta|^{n_{1}}+|\delta|^{n_{2}}+|\delta|^{n_{3}}<3,
$$

which is false for $\ell \geq 1$ and $d \geq 1$. Therefore, $\Lambda_{1} \neq 0$.

So we apply Theorem 3.1 with the data

$$
\begin{gathered}
t:=3, \quad \eta_{1}:=10, \quad \eta_{2}:=\gamma, \quad \eta_{3}:=\frac{4 d \sqrt{2}}{9\left(1+\gamma^{n_{1}-n_{3}}+\gamma^{n_{2}-n_{3}}\right)}, \\
b_{1}:=\ell, \quad b_{2}:=-n_{3}, \quad b_{3}:=1 .
\end{gathered}
$$

Since $\eta_{1}, \eta_{2}, \eta_{2} \in \mathbb{Q}(\sqrt{2})$, we take the field $\mathbb{K}:=\mathbb{Q}(\sqrt{2})$ with degree $D_{\mathbb{K}}:=2$. Since $\max \left\{1, \ell, n_{3}\right\} \leq$ $n_{1}$, we take $B:=n_{1}$. The minimal polynomial of $\gamma$ over $\mathbb{Z}$ is $x^{2}-6 z+1$ has roots $\gamma$ and $\delta$ with 
$5<\gamma<6$ and $0<\delta<1$. Thus, we can take $h(\gamma)=\frac{1}{2} \log \gamma, h(10)=\log 10$, and $h(\sqrt{2})=\frac{1}{2} \log 2$. Also,

$$
\begin{aligned}
h\left(\eta_{3}\right) & \leq h(4 d \sqrt{2})+h\left(9\left(\left(1+\gamma^{n_{1}-n_{3}}+\gamma^{n_{2}-n_{3}}\right)\right)\right) \\
& \leq h(4)+h(d)+h(\sqrt{2})+h(9)+h\left(\left(1+\gamma^{n_{1}-n_{3}}+\gamma^{n_{2}-n_{3}}\right)\right) \\
& \leq h(4)+h(d)+h(\sqrt{2})+h(9)+h(1)+\left(\left(n_{1}-n_{2}\right)+\left(n_{2}-n_{3}\right)\right) h(\gamma)+2 \log 2 \\
& \leq \frac{9}{2} \log 2+4 \log 3+\frac{1}{2}\left(n_{1}-n_{3}\right) \log \gamma \\
& \leq 8+\frac{1}{2}\left(n_{1}-n_{3}\right) \log \gamma .
\end{aligned}
$$

Thus, we can take $A_{1}:=2 \log 10, A_{2}:=\log \gamma$, and $A_{3}:=16+\left(n_{1}-n_{3}\right) \log \gamma$. So, Theorem 3.1 tells us that the left-hand side of (4.4) is bounded below by

$$
\begin{aligned}
\log \left|\Lambda_{1}\right|> & -1.4 \times 30^{6} \times 3^{4.5} \times 2^{2}(1+\log 2)\left(1+\log n_{1}\right) \\
& \times(2 \log 10)(\log \gamma)\left(16+\left(n_{1}-n_{3}\right) \log \gamma\right) \\
> & -4.37 \times 10^{14}\left(n_{1}-n_{3}\right) \log n_{1} .
\end{aligned}
$$

By comparing the above inequality with the right-hand side of (4.4) we get that

$$
n_{1} \leq 2.48 \times 10^{14}\left(n_{1}-n_{3}\right) \log n_{1}
$$

4.2. Step 2. We have that

$$
\frac{\gamma^{n_{1}}-\delta^{n_{1}}}{4 \sqrt{2}}+\frac{\gamma^{n_{2}}-\delta^{n_{2}}}{4 \sqrt{2}}-\frac{d \cdot 10^{\ell}}{9}=-\frac{d}{9}-\frac{\gamma^{n_{3}}-\delta^{n_{3}}}{4 \sqrt{2}}
$$

This is equivalent to

$$
\frac{\gamma^{n_{1}}}{4 \sqrt{2}}\left(1+\gamma^{n_{2}-n_{1}}\right)-\frac{d \cdot 10^{\ell}}{9}=-\frac{d}{9}-\frac{\gamma^{n_{3}}}{4 \sqrt{2}}+\frac{1}{4 \sqrt{2}}\left(\delta^{n_{1}}+\delta^{n_{2}}+\delta^{n_{3}}\right) .
$$

Thus, we can conclude that

$$
\begin{aligned}
\left|\frac{\gamma^{n_{1}}}{4 \sqrt{2}}\left(1+\gamma^{n_{2}-n_{1}}\right)-\frac{d \cdot 10^{\ell}}{9}\right| & \leq \frac{d}{9}+\frac{\gamma^{n_{3}}}{4 \sqrt{2}}+\frac{1}{4 \sqrt{2}}\left(\delta^{n_{1}}+\delta^{n_{2}}+\delta^{n_{3}}\right) \\
& \leq 1+\frac{\gamma^{n_{3}}}{4 \sqrt{2}}+\frac{3}{4 \sqrt{2}}=\frac{3+4 \sqrt{2}+\gamma^{n_{3}}}{4 \sqrt{2}} \\
& <\frac{\gamma^{n_{3}+1}}{4 \sqrt{2}} .
\end{aligned}
$$

Thus, we have

$$
\left|\frac{\gamma^{n_{1}}}{4 \sqrt{2}}\left(1+\gamma^{n_{2}-n_{1}}\right)-\frac{d \cdot 10^{\ell}}{9}\right|<\frac{\gamma^{n_{3}+1}}{4 \sqrt{2}} .
$$

Dividing through 4.6 by $\alpha^{n_{2}}\left(1+\gamma^{n_{1}-n_{2}}\right) /(4 \sqrt{2})$, we get

$$
\left|10^{\ell} \gamma^{-n_{2}}\left(\frac{4 d \sqrt{2}}{9\left(1+\gamma^{n_{1}-n_{2}}\right)}\right)-1\right|<\frac{\gamma^{n_{3}-n_{2}+1}}{1+\gamma^{n_{1}-n_{2}}}<\gamma^{n_{3}-n_{2}+1} .
$$

We put

$$
\Lambda_{2}:=10^{\ell} \gamma^{-n_{2}}\left(\frac{4 d \sqrt{2}}{9\left(1+\gamma^{n_{1}-n_{2}}\right)}\right)-1
$$


As before, in order to apply Theorem 3.1 we need to check that $\Lambda_{2} \neq 0$. Suppose that $\Lambda_{2}=0$, then we have

$$
\gamma^{n_{1}}+\gamma^{n_{2}}=\frac{10^{\ell} \cdot 4 d \sqrt{2}}{9} .
$$

By conjugating the above relation in $\mathbb{Q}(\sqrt{2})$, we get

$$
\delta^{n_{1}}+\delta^{n_{2}}=-\frac{10^{\ell} \cdot 4 d \sqrt{2}}{9} .
$$

Taking absolute values on both sides of the above equation, we obtain that

$$
\frac{10^{\ell} \cdot 4 d \sqrt{2}}{9}=\left|\delta^{n_{1}}+\delta^{n_{2}}\right| \leq|\delta|^{n_{1}}+|\delta|^{n_{2}}<2
$$

which is false for $\ell \geq 1$ and $d \geq 1$. Therefore, $\Lambda_{2} \neq 0$. So we apply Theorem 3.1 with the data

$$
t:=3, \quad \eta_{1}:=10, \quad \eta_{2}:=\gamma, \quad \eta_{3}:=\frac{4 d \sqrt{2}}{9\left(1+\gamma^{n_{1}-n_{2}}\right)}, \quad b_{1}:=\ell, \quad b_{2}:=-n_{2}, \quad b_{3}:=1 .
$$

We take $D:=2, B:=n_{1}, A_{1}:=2 \log 10, A_{2}:=\log \gamma$ and

$$
\begin{aligned}
A_{3}:=2 h\left(\eta_{3}\right) & =2\left(h(4 d \sqrt{2})+h(9)+h\left(1+\gamma^{n_{1}-n_{2}}\right)\right) \\
& \leq 2\left(h(4)+h(d)+h(\sqrt{2})+h(9)+\left(n_{1}-n_{2}\right) h(\gamma)+\log 2\right) \\
& \leq 14+\left(n_{1}-n_{2}\right) \log \gamma .
\end{aligned}
$$

So, Theorem 3.1 , tells us that

$$
\begin{aligned}
\log \left|\Lambda_{2}\right|> & -1.4 \times 30^{6} \times 3^{4.5} \times 2^{2}(1+\log 2)\left(1+\log n_{1}\right)(2 \log 10) \\
& \times(\log \gamma)\left(14+\left(n_{1}-n_{2}\right) \log \gamma\right) \\
> & -2.48 \times 10^{14}\left(n_{1}-n_{2}\right) \log n_{1} .
\end{aligned}
$$

By comparing the above ineqguality with 4.7 , we conclude that

$$
n_{2}-n_{3}<1.42 \times 10^{14}\left(n_{1}-n_{2}\right) \log n_{1} .
$$

4.3. Step 3. We have that

$$
\frac{\gamma^{n_{1}}-\delta^{n_{1}}}{4 \sqrt{2}}-\frac{d \cdot 10^{\ell}}{9}=-\frac{d}{9}-\frac{\gamma^{n_{2}}-\delta^{n_{2}}}{4 \sqrt{2}}-\frac{\gamma^{n_{3}}-\delta^{n_{3}}}{4 \sqrt{2}} .
$$

This is equivalent to

$$
\frac{\gamma^{n_{1}}}{4 \sqrt{2}}-\frac{d \cdot 10^{\ell}}{9}=-\frac{d}{9}-\frac{\gamma^{n_{2}}}{4 \sqrt{2}}-\frac{\gamma^{n_{3}}}{4 \sqrt{2}}+\frac{1}{4 \sqrt{2}}\left(\delta^{n_{1}}+\delta^{n_{2}}+\delta^{n_{3}}\right) .
$$

Thus, we can conclude that

$$
\begin{aligned}
\left|\frac{\gamma^{n_{1}}}{4 \sqrt{2}}-\frac{d \cdot 10^{\ell}}{9}\right| & \leq \frac{d}{9}+\frac{\gamma^{n_{2}}}{4 \sqrt{2}}+\frac{\gamma^{n_{3}}}{4 \sqrt{2}}+\frac{1}{4 \sqrt{2}}\left(\delta^{n_{1}}+\delta^{n_{2}}+\delta^{n_{3}}\right) \\
& \leq 1+\frac{\gamma^{n_{2}}+\gamma^{n_{3}}}{4 \sqrt{2}}+\frac{3}{4 \sqrt{2}}=\frac{3+4 \sqrt{2}+\gamma^{n_{2}}+\gamma^{n_{3}}}{4 \sqrt{2}} \\
& <\frac{\gamma^{n_{2}+2}}{4 \sqrt{2}} .
\end{aligned}
$$

Thus, we have

$$
\left|\frac{\gamma^{n_{1}}}{4 \sqrt{2}}-\frac{d \cdot 10^{\ell}}{9}\right|<\frac{\gamma^{n_{2}+2}}{4 \sqrt{2}}
$$


Dividing through 4.9 by $\alpha^{n_{1}} /(4 \sqrt{2})$, we get

$$
\left|10^{\ell} \alpha^{-n_{1}}\left(\frac{4 d \sqrt{2}}{9}\right)-1\right|<\alpha^{n_{2}-n_{1}+2} .
$$

We put

$$
\Lambda_{3}=10^{\ell} \alpha^{-n_{1}}\left(\frac{4 d \sqrt{2}}{9}\right)-1 .
$$

As in the previous cases, in order to apply Theorem 3.1 we need to check that $\Lambda_{3} \neq 0$. Suppose that $\Lambda_{3}=0$, then we have

$$
\gamma^{n_{1}}=\frac{10^{\ell} \cdot 4 d \sqrt{2}}{9}
$$

By conjugating the above relation in $\mathbb{Q}(\sqrt{2})$, we get

$$
\delta^{n_{1}}=-\frac{10^{\ell} \cdot 4 d \sqrt{2}}{9} .
$$

Taking absolute values on both sides of the above equation, we obtain that

$$
\frac{10^{\ell} \cdot 4 d \sqrt{2}}{9}=\left|\delta^{n_{1}}\right| \leq|\delta|^{n_{1}}<1,
$$

which is false for $\ell \geq 1$ and $d \geq 1$. Thus, $\Lambda_{3} \neq 0$. So, we apply Theorem 3.1 with the data

$$
t:=3, \quad \eta_{1}:=10, \quad \eta_{2}:=\gamma, \quad \eta_{3}:=\frac{4 d \sqrt{2}}{9}, \quad b_{1}:=\ell, \quad b_{2}:=-n_{2}, \quad b_{3}:=1 .
$$

We take $D:=2, B:=n_{1}, A_{1}:=2 \log 10, A_{2}:=\log \gamma$ and $A_{3}:=2 h\left(\eta_{3}\right) \leq 11$. Theorem 3.1. tells us that

$$
\begin{aligned}
\log \left|\Lambda_{3}\right| & >\quad-1.4 \times 30^{6} \times 3^{4.5} \times 2^{2}(1+\log 2)\left(1+\log n_{1}\right)(2 \log 10)(\log \gamma) \times 11 \\
& >-1.73 \times 10^{14} \log n_{1} .
\end{aligned}
$$

By comparing this with 4.10 , we obtain that

$$
n_{1}-n_{2}<9.8 \times 10^{13} \log n_{1} .
$$

Now we combine the inequalities (4.5), 4.8) and 4.11) to obtain the bound on $n_{1}$ as follows:

$$
\begin{aligned}
n_{1} & <2.48 \times 10^{14}\left(1.42 \times 10^{14}\left(9.8 \times 10^{13} \log n_{1}\right) \log n_{1}\right) \log n_{1} \\
& <3.5 \times 10^{42}\left(\log n_{1}\right)^{3} .
\end{aligned}
$$

Now, we apply Lemma 3.2 on the above inequality 4.12 with the data: $r:=3, H:=3.5 \times$ $10^{42}, L:=n_{1}$. We obtain that $n_{1}<2.6 \times 10^{49}$. We record what we have proved.

Lemma 4.1. Let $\left(N, n_{1}, n_{2}, n_{3}, d, \ell\right)$ be the nonnegative integer solutions to the equation 2.1) with $n_{1} \geq n_{2} \geq n_{3} \geq 0,1 \leq d \leq 9$, and $\ell \geq 1$. Then we have

$$
\ell<n_{1}<10^{50} \text {. }
$$




\section{REDUCING THE BOUNDS}

The bounds ontained in Lemma 4.1 are too large to carry out meaningful computations on the computer. Thus, we need to reduce these bounds. To do so, we return to 4.4, 4.7), and 4.10) and apply Lemma 3.1 via the following procedure.

First, we put

$$
\Gamma_{3}:=\ell \log 10-n_{1} \log \gamma+\log \left(\frac{4 d \sqrt{2}}{9}\right), \quad 1 \leq d \leq 9 .
$$

For technical reasons, we assume that $n_{1}-n_{2} \geq 20$ for the moment and go to 4.10 . We will discuss how to get rid of this condition later. Note that $e^{\Gamma_{3}}-1=\Lambda_{3} \neq 0$. Thus, $\Gamma_{3} \neq 0$. If $\Gamma_{3}<0$ then

$$
0<\left|\Gamma_{3}\right|<e^{\left|\Gamma_{3}\right|}-1=\left|\Lambda_{3}\right|<\frac{1}{\gamma^{n_{1}-n_{2}-2}} .
$$

If $\Gamma_{3}>0$, then we have that $\left|e^{\Gamma_{3}}-1\right|<1 / 2$. Hence $e^{\Gamma_{3}}<2$. Thus, we get that

$$
0<\Gamma_{3}<e^{\Gamma_{3}}-1=e^{\Gamma_{3}}\left|\Lambda_{3}\right|<\frac{2}{\gamma^{n_{1}-n_{2}-2}} .
$$

Therefore, in both cases, we have that

$$
0<\left|\Gamma_{3}\right|=\left|\ell \log 10-n_{1} \log \gamma+\log \left(\frac{4 d \sqrt{2}}{9}\right)\right|<\frac{2 \gamma^{2}}{\gamma^{n_{1}-n_{2}}} .
$$

Dividing through the above inequality by $\log \gamma$, we get

$$
0<\left|\ell \frac{\log 10}{\log \gamma}-n_{1}+\frac{\log ((4 d \sqrt{2}) / 9)}{\log \gamma}\right|<\frac{2 \gamma^{2}}{\gamma^{n_{1}-n_{2}} \log \gamma}
$$

If we put

$$
\tau:=\frac{\log 10}{\log \gamma} \quad \text { and } \quad \mu_{d}:=\frac{\log ((4 d \sqrt{2}) / 9)}{\log \gamma}, \quad 1 \leq d \leq 9,
$$

we can rewrite (5.1) as

$$
0<\left|\ell \tau-n_{1}+\mu_{d}\right|<39 \cdot \gamma^{-\left(n_{1}-n_{2}\right)} .
$$

We now apply Lemma 3.1 on 5.2 . We put $M:=10^{50}$. A quick computer search in Mathematica reveals that the convergent

$$
\frac{p_{108}}{q_{108}}=\frac{2703843740443108411802421359516257223259008405220106}{2069931281589203990595364033267574277731383243231951}
$$

of $\tau$ is such that $q_{108}>6 M$ and $\varepsilon_{d} \geq 0.0623392>0$. Therefore, with $A:=46$ and $B:=\gamma$ we calculated each value of $\log \left(39 q_{108} / \varepsilon_{d}\right) / \log \gamma$ and found that all of them are at most 70 . Thus, we have that $n_{1}-n_{2} \leq 70$. In the case that $n_{1}-n_{2}<20$, we would have that $n_{1}-n_{2}<20<70$. Thus, $n_{1}-n_{2}<70$ holds in both cases.

Next, we put

$$
\Gamma_{2}:=\ell \log 10-n_{2} \log \gamma+\log \left(\frac{4 d \sqrt{2}}{9\left(1+\gamma^{n_{1}-n_{2}}\right)}\right), \quad 1 \leq d \leq 9 .
$$

For technical reasons, as before we assume that $n_{2}-n_{3} \geq 20$ for the moment and go to (4.7). We will discuss how to get rid of this condition later. Note that $e^{\Gamma_{2}}-1=\Lambda_{2} \neq 0$. Thus, $\Gamma_{2} \neq 0$. If 
$\Gamma_{2}<0$, then

$$
0<\left|\Gamma_{2}\right|<e^{\left|\Gamma_{2}\right|}-1=\left|\Lambda_{2}\right|<\frac{1}{\gamma^{n_{2}-n_{3}-1}} .
$$

If $\Gamma_{2}>0$, then we have that $\left|e^{\Gamma_{2}}-1\right|<1 / 2$. Hence $e^{\Gamma_{2}}<2$. Thus, we get that

$$
0<\Gamma_{2}<e^{\Gamma_{2}}-1=e^{\Gamma_{2}}\left|\Lambda_{2}\right|<\frac{2}{\gamma^{n_{2}-n_{3}-1}} .
$$

Therefore, in both cases, we have that

$$
0<\left|\Gamma_{2}\right|=\left|\ell \log 10-n_{1} \log \gamma+\log \left(\frac{4 d \sqrt{2}}{9\left(1+\gamma^{n_{1}-n_{2}}\right)}\right)\right|<\frac{2 \gamma}{\gamma^{n_{2}-n_{3}}} .
$$

Dividing through the above inequality by $\log \gamma$, we get

$$
0<\left|\ell \frac{\log 10}{\log \gamma}-n_{2}+\frac{\log \left((4 d \sqrt{2}) / 9\left(1+\gamma^{n_{1}-n_{2}}\right)\right)}{\log \gamma}\right|<\frac{2 \gamma}{\gamma^{n_{2}-n_{3}} \log \gamma} .
$$

We put

$$
\tau:=\frac{\log 10}{\log \gamma} \quad \text { and } \quad \mu_{d, k}:=\frac{\log \left((4 d \sqrt{2}) / 9\left(1+\gamma^{k}\right)\right)}{\log \gamma}, \quad 1 \leq d \leq 9, \quad 1 \leq k \leq 70,
$$

where $k:=n_{1}-n_{2}$. We can rewrite 5.3 as

$$
0<\left|\ell \tau-n_{2}+\mu_{d, k}\right|<7 \cdot \gamma^{-\left(n_{2}-n_{3}\right)} .
$$

We now apply Lemma 3.1 on (5.4). We put $M:=10^{50}$. A quick computer search in Mathematica reveals that the 108-th convergent of $\tau$ is such that $q_{108}>6 M$ and $\varepsilon_{d, k} \geq 0.00139047>0$. Therefore, with $A:=7$ and $B:=\gamma$ we calculated each value of $\log \left(7 q_{108} / \varepsilon_{d, k}\right) / \log \gamma$ and found that all of them are at most 72 . Thus, we have that $n_{2}-n_{3} \leq 72$. In the case that $n_{2}-n_{3}<20$, we would have that $n_{2}-n_{3}<20<72$. Thus, $n_{2}-n_{3} \leq 72$ holds in both cases.

Lastly, we put

$$
\Gamma_{1}:=\ell \log 10-n_{3} \log \gamma+\log \left(\frac{4 d \sqrt{2}}{9\left(1+\gamma^{n_{1}-n_{3}}+\gamma^{n_{2}-n_{3}}\right)}\right), \quad 1 \leq d \leq 9 .
$$

We use the original assumption that $n_{1}>100$ and go to 4.4. Note that $e^{\Gamma_{1}}-1=\Lambda_{1} \neq 0$. Thus, $\Gamma_{1} \neq 0$. If $\Gamma_{1}<0$, then

$$
0<\left|\Gamma_{1}\right|<e^{\left|\Gamma_{1}\right|}-1=\left|\Lambda_{1}\right|<\frac{1}{\gamma^{n_{1}-2}} .
$$

If $\Gamma_{1}>0$, then we have that $\left|e^{\Gamma_{1}}-1\right|<1 / 2$. Hence $e^{\Gamma_{1}}<2$. Thus, we get that

$$
0<\Gamma_{1}<e^{\Gamma_{1}}-1=e^{\Gamma_{1}}\left|\Lambda_{1}\right|<\frac{2}{\gamma^{n_{1}-2}} .
$$

Therefore, in both cases, we have that

$$
0<\left|\Gamma_{1}\right|=\left|\ell \log 10-n_{3} \log \gamma+\log \left(\frac{4 d \sqrt{2}}{9\left(1+\gamma^{n_{1}-n_{2}}+\gamma^{n_{2}-n_{3}}\right)}\right)\right|<\frac{2 \gamma^{2}}{\gamma^{n_{1}}} .
$$

Dividing through the above inequality by $\log \gamma$, we get

$$
0<\left|\ell \frac{\log 10}{\log \gamma}-n_{3}+\frac{\log \left((4 d \sqrt{2}) / 9\left(1+\gamma^{n_{1}-n_{3}}+\gamma^{n_{2}-n_{3}}\right)\right)}{\log \gamma}\right|<\frac{2 \gamma^{2}}{\gamma^{n_{1}} \log \gamma} .
$$


We put

$$
\tau:=\frac{\log 10}{\log \gamma} \quad \text { and } \quad \mu_{d, k, s}:=\frac{\log \left((4 d \sqrt{2}) / 9\left(1+\gamma^{k}+\gamma^{s}\right)\right)}{\log \gamma}, \quad 1 \leq d \leq 9,
$$

where $1 \leq k:=n_{1}-n_{3}=\left(n_{1}-n_{2}\right)+\left(n_{2}-n_{3}\right) \leq 142$ and $1 \leq s:=n_{2}-n_{3} \leq 72$. We can rewrite (5.5) as

$$
0<\left|\ell \tau-n_{3}+\mu_{d, k, s}\right|<39 \cdot \gamma^{-n_{1}} .
$$

We again apply Lemma 3.1 on $(5.6)$. We also put $M:=10^{50}$. A quick computer search in Mathematica reveals that the 108-th convergent of $\tau$ is such that $q_{108}>6 M$ and $\varepsilon_{d, k, s} \geq 0.00125>$ 0 . Therefore, with $A:=39$ and $B:=\gamma$ we calculated each value of $\log \left(39 q_{108} / \varepsilon_{d, k, s}\right) / \log \gamma$ and found that all of them are at most 76 . Thus, we have that $n_{1} \leq 76$. This contradicts our assumption that $n_{1}>100$. Hence, Theorem 2.1 holds.

Acknowledgement. The author thanks the referee for the useful comments and suggestions that greatly improved the quality of presentation of the paper.

\section{REFERENCES}

[1] Bahera, A. - PAnda, G. K.: On the square roots of triangular numbers. The Fibonacci Quarterly, 37(2):98$105,1999$.

[2] Bugeaud, Y. - Mignotte, M. - Siksek, S.: Classical Classical and modular approaches to exponential Diophantine equations I. Fibonacci and Lucas perfect powers. Annals of Mathematics, 163(2):969-1018, 2006.

[3] Dujella, A. - Ретнö, A.: A generalization of a theorem of Baker and Davenport. The Quarterly Journal of Mathematics, 49(195):291-306, 1998.

[4] Gúzman SÁnchez, S. - LuCA, F.: Linear combinations of factorials and s-units in a binary recurrence sequence. Annales Mathemántiques du Québec, 38(2):169-188, 2014.

[5] García Lomelí, A. C. - Hernández Hernández, S.: Repdigits as sums of two Padovan numbers. Journal of Integer Sequences, 22(2): Article 19.2.3, 2019.

[6] LuCA, F.: Repdigits as sums of three Fibonacci numbers. Math. Commun., 17:1-11, 2012.

[7] Luca, F. - Normenyo, B. V. - Togbé, A.: Repdigits as sums of three Lucas numbers. Colloquium Mathematicum, 156(2):255-265, 2019.

[8] Matveev, E. M.: An explicit lower bound for a homogeneous rational linear form in the logarithms of algebraic numbers II. Izv. Ross. Akad. Nauk Ser. Mat., 64(6):125-180, 2000, in Russian: English translation in Izvestiya Mathematics, 64(6):1217-1269, 2000.

[9] Normenyo, B. V. - LucA, F. - Togbé, A.: Repdigits as sums of three Pell numbers. Periodica Mathematica Hungarica, $\mathbf{7 7}(2): 318-328,2018$.

[10] OEIS Foundation Inc.: The On-Line Encyclopedia of Integer Sequences, https://oeis.org 2019.

Institute of Analysis and Number Theory

Graz University of Technology

Kopernikusgasse 24/II

A-8010 GraZ

AUSTRIA

E-mail address: mddamulira@tugraz.at; mahadi@aims.edu.gh 